\title{
3-D Structure of Z-disks isolated from the flight muscle of Lethocerus indicus
}

Fatemeh Abbasi Yeganeh ${ }^{1}$, Hamidreza Rahmani ${ }^{1}$, Kenneth Taylor ${ }^{2}$ and Dianne Taylor ${ }^{1}$

${ }^{1}$ florida state university, United States, ${ }^{2}$ Florida State University, Florida, United States

The recent publication of a 3-D image of isolated Z-disks from Apis mellifera, honey-bee, flight muscle [Rusu et al., J. Muscle Res Cell Motil (2017)] used the relatively harsh treatment of high KCl/KI to remove both thick and thin filaments in a single step that nevertheless produced a specimen suitable for cryoelectron tomography (cryoET). The reconstruction, the first of its kind utilizing isolated Z-disks, confirmed many details of the previously 3-D image obtained from plastic sections. Here we use a different approach for Z-disk isolation from, in this case, flight muscle from the large waterbug Lethocerus indicus that combines $1.4 \mathrm{M} \mathrm{NaCl}$ and $10 \mathrm{mM}$ pyrophosphate to remove the thick filaments and calcium in sensitive gelsolin for $4.5 \mathrm{~min}$ to remove the thin filaments from the I-Z-I brushes. Tilt series are collected on a Titan Krios electron microscope equipped with a DE-20 camera and merged using PROTOMO. By adding more tiltseries to our previous analysis we are improving the resolutions. We conclude that Z-disks from Apis mellifera and Lethocerus indicus are similar but not identical by comparing the Fourier transforms. Fourier transforms of the Z-disks show spots from a hexagonal lattice with a spacing of $520 \AA$ extending to $87 \AA$ and Fourier transform of cryo-EM micrograph of a Lethocerus Z-disc extends to 6 orders. The large solvent channel of honey bee is not as prominent in Lethocerus Zdisks and this is reflected in the 1,0 spots in the Lethocerus transform are weak and nearly absent, whereas the corresponding spots in the honey bee transform are strong. There is ample evidence that the actin filaments in the overlap zone of both bee and waterbug flight muscle present a helical array of target zones around each thick filament. However, Squire [SQUIRE, J. M. (1972) J. molec. Biol. 72, 125-38] has pointed out that Cheng and Deatherage's [Cheng, N., and J. E Deatherage. (1989). J. Cell Biol. 108:17611774] 3D reconstruction of the honey bee Z-band, when extrapolated in to the A-band, implies rings of target zones rather than helices of target zones surrounding the thick filament. This discrepancy might be resolved if there are there are three fold screw axes at the lattice and trigonal positions of the of the insect Z-band instead of three-fold rotation axes; thus making the insect Z-band symmetry P $3{ }_{1} 21$, instead of the P312 symmetry assumed by C\&D.

Supported by NIH and AHA.
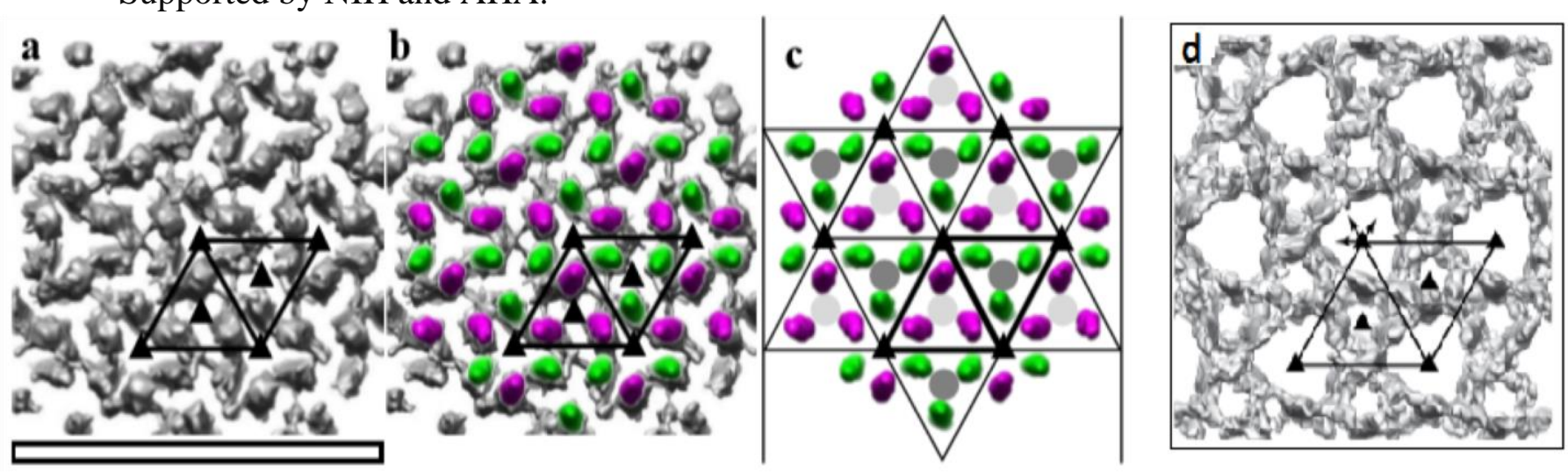

Figure 1. . Density Map of Global Averages of Lethocerus Z-disc. Increasing the density of the symmetrized global average revealed the unique network of connecting densities that maintains the arrangement of the thin filaments of opposite and same orientations. The thin filament connections form two different channels: the large channel formed by the thin filaments at the 
apexes of each unit cell that are formed by six thin filaments of altering orientation, and the two small solvent channels within the unit cell that are formed by three thin filaments of the same polarity. a) Density map (grey) of a symmetrized global average showing the connecting densities that form the Z-Disc. b) Overlap of thin filaments from Fig 5 (purple and green) on the density map. c) The p321 symmetry that is seen in the thin filament arrangement. The dark and light grey circles represent the positions of the thick filaments in the A-band of adjacent sarcomeres. The unit cell in all the images are represented with thick black lines. White scale bar $=155 \mathrm{~nm}$. d) Density map showing the connecting densities that form the Z-Disc from Apis[1] showing bigger channels compared to Lethocerus.

i.
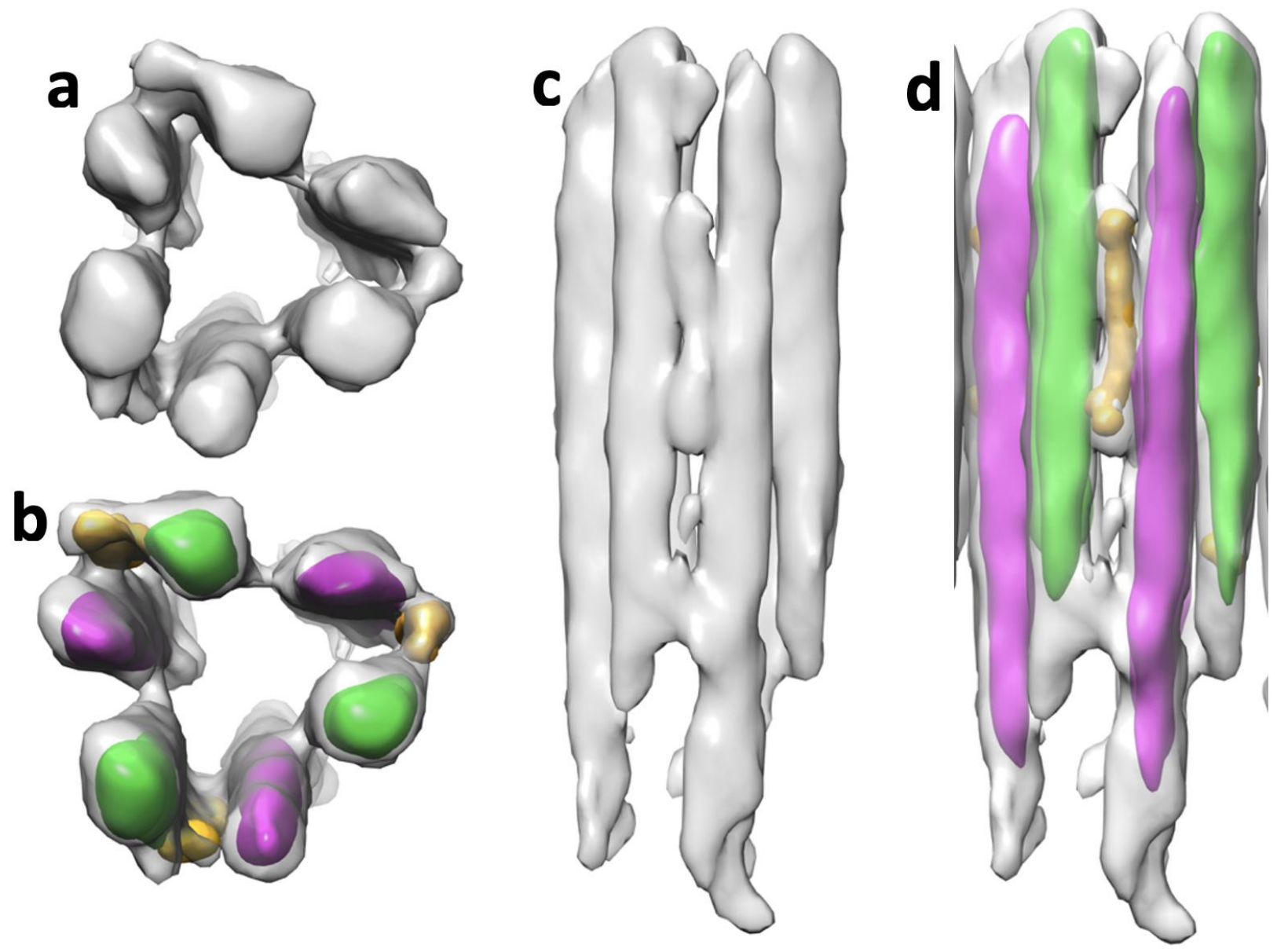

Figure 2. Showing the large solvent channel within the Z-disk and the $\alpha$-actinin placed in the $\mathrm{C} 1$ connecting density. a) Large solvent channel density map(top view. b) (a) with the $\mathrm{C} 1$ - $\alpha$-actinin (gold) found at $\mathrm{t}$ h e apexes of the big triangle between actin filaments of opposite orientations (green and purple). c) Side vi e w of (a), $\mathrm{C} 1$ is the shorter density between the longer actin densities. d) Side view of (b).

\section{References}

1.Rusu et al., J. (2017).J. Muscle Res Cell Motil 38:241-250.

2.Cheng, N., and J E Deatherage. (1989). J. Cell Biol. 108:1761-1774.

3.Cope, J., Heuman.n, J., \& Hoenger, A. (2011). Curr Protoc Protein Sci,ch 17,Unit17.13.

4.REEDY, M. K. (1968) J. molec. Biol. 31, 155-76.

5.SQUIRE, J. M. (1972) J. molec. Biol. 72, 125-38.

6.Thach, R.E., \& Thach, S.S. (1971). Biophys J., 11, 204-210. 